\title{
Editorial
}

\section{La mejor política económica es la del bien común}

Lo que comenzó siendo una discusión técnica entre asesores extranjeros del Ministerio de Hacienda para elevar la recaudación fiscal del gobierno se convirtió en cuatro medidas económicas que el gobierno presenta como plan. El presidente de la república se refirió a las medidas como "una plataforma para el desarrollo social porque busca el bienestar y el progreso de todos los salvadoreños, no de grupos", pero casi unánimemente, todos los sectores sociales, menos las grandes gremiales de la empresa privada, las rechazaron.

Exceptuando al gran capital, los otros sectores de la economía nacional consideran que las propuestas gubernamentales son inconsistentes, injustificadas y contradictorias en sí mismas, con las políticas anteriores y con los objetivos declarados que dicen pretender. En los artículos y comentarios de esta edición de la revista ECA se analizan y discuten en detalle dichas medidas. Aquí queremos reflexionar sobre sus implicaciones sociales desde la perspectiva de las mayorías populares y del bien común.

\section{Los propósitos declarados versus las realidades}

Los propósitos declarados de la nueva política económica son (a) transformar el crecimiento económico en un instrumento para un desarrollo integral, sostenible, sustentable y equitativo; (b) eliminar las distorsiones que benefician a unos pocos en perjuicio de las grandes mayorías; (c) mejorar las condiciones de vida en las áreas rurales para reducir la emigración a las ciudades y en las áreas urbanas para erradicar la pobreza concentrada en sus cordones marginales; (d) fortalecer a la micro y pequeña empresa; (e) hacer atractivo el país a la inversión extranjera e incorporarlo a las cadenas de producción mundial "para que todos vivamos mejor, especialmente nuestros trabajado- 


\section{El crecimiento nacional, uno de los logros de los cuales se vanaglorían los gobernantes de ARENA y una de las premisas fundamentales de la nueva política económica, es ficticio.}

res" (Discurso del presidente de la república, 2 de febrero de 1995).

Para conseguir estos objetivos, el gobierno propone cuatro medidas: establecer un tipo de cambio fijo que incluye la desaparición del Banco Central de Reserva, disminuir gradualmente los aranceles, modernizar el Estado - pero sobre todo privatizar las empresas estatales existentes-, e incrementar la recaudación fiscal para evitar la evasión de más del 50 por ciento - sin descartar el incremento del impuesto al valor agregado en el futuro.

El debate suscitado alrededor de las medidas obligó a reflexionar como nunca antes se habia hecho sobre las posibilidades y debilidades de la economía nacional. En esta discusión apareció con claridad meridiana que el crecimiento nacional, uno de los logros de los cuales se vanaglorian los gobernantes de ARENA y una de las premisas fundamentales de la nueva política económica, es ficticio. Ni siquiera se puede decir que sea resultado del mercado, puesto que más bien es producto de la monetarización de las remesas familiares a las cuales "artificiosamente" se las llama "divisas". El "enigma" del crecimiento económico actual consiste en que el sacrificio de los emigrados pobres residentes en Estados Unidos sostiene el mercado en El Salvador -otra de las premisas básicas del bienestar-, pero éste no sostiene ni refuerza a los pobres (ver el artículo del Departamento de Economía). La tasa de crecimiento real que favorece a unos pocos, va acompañada de una tasa de inflación mayor que castiga a la mayoría. Por lo tanto, el crecimiento de la economía salvadoreña discrimina cada vez más los ingresos.

En la polémica aparece también con bastante claridad que las medidas propuestas no responden al estado real de la economía salvadoreña. Todavía existen muchas fisuras en los ejes productivos y el Estado no está preparado para ayudar a cerrarlas. Una apertura externa como la propuesta requiere de una integración económica interna, de una diversificación y de una modemización mayores. Antes de abrir la economía salvadoreña en los términos propuestos, es necesario superar varios puntos críticos, en concreto, la desarticulación existente entre la producción agropecuaria y la industrial y los desequilibrios en los salarios, la inversión y los impuestos indirectos. Estos puntos críticos explican que la economía salvadoreña se dedique predominantemente a los servicios y al comercio sin producir.

Prescindiendo de si la empresa privada utilizó bien o no los subsidios gubernamentales para reconvertirse y volverse competitiva, aspecto que 
requiere de un análisis técnico riguroso, es claro que los grandes bloques económicos como la Unión Europea, Japón y Estados Unidos, Canadá y México se protegen de la competencia externa, pese a sus discursos sobre las bondades del mercado libre. Esto quiere decir que la producción nacional no encuentra fácilmente el camino hacia el mercado internacional y si no se puede exportar a otros mercados sirve de muy poco producir. No obstante la doctrina neoliberal sobre la libertad del mercado, los

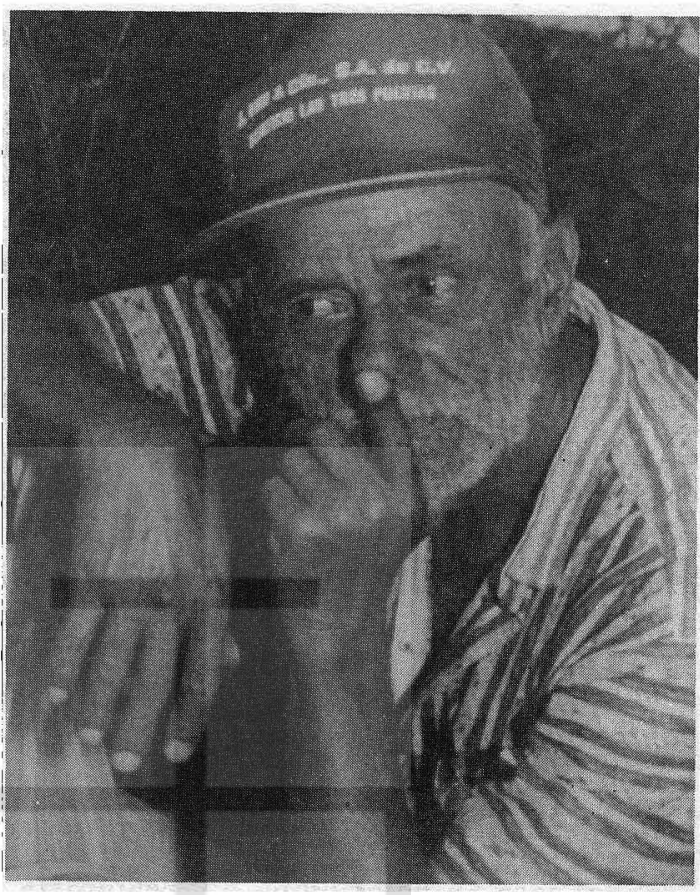
grandes bloques tienden a proteger sus mercados regionales con medidas extra económicas. Incluso la maquila, de la cual el gobierno quiere: hacer una panacea, tiene sus límites, pues está sometida a cuotas de importación.

Ante estas realidades del orden económico internacional, la posibilidad de que El Salvador o Centroamérica sean incluidas en el tratado de libre comercio de Norteamérica son muy remotas. Por lo tanto, la responsabilidad no es única y exclusivamente de los productores y exportadores salvadoreños. De ahí que la sobrevivencia de países como el nuestro dependa en mucho mayor medida que la de los países industrializados de la conformación de un bloque regional con poder para negociar la apertura de los mercados internos con otros bloques. Entre mayor sea el poder de negociación, mayor es la posibilidad para entrar y competir exitosamente en los mercados de los otros bloques.

Es incomprensible, por tanto, que El Salvador abandone la integración regional para lanzarse sola a una aventura, cuyo éxito no está asegurado. En lugar de abrir totalmente sus fronteras sin recibir nada a cambio y de abandonar por las buenas su limitado poder de negociación, El Salvador debiera impulsar con más interés y energía la integración del bloque centroamericano. Esta es la única manera viable para expandir el reducido mercado regional.

Si la política salvadoreña impacta negativamente las otras economías 
centroamericanas, es natural que éstas busquen como protegerse, pudiendo llegar hasta el extremo de romper los pactos regionales acordados. Los defensores de la apertura total quieren engañarse y engañar a la opinión pública, presentando a El Salvador como el país centroamericano más avanzado en la carrera hacia la modernización. Recurriendo al nacionalismo supenficial, pretenden convencer de que El Salvador se ha vuelto un ejemplo de política económica y el líder de la modernidad en el área. Por lo tanto, a los otros países centroamericanos no les quedaría otra alternativa que subirse al tren del progreso impulsado por El Salvador o hundirse para siempre en el subdesarrollo. Para quienes así piensan, Centroamérica se habría convertido en un peso intolerable para El Salvador. La verdad es muy otra, pues nuestro país no está preparado para asumir los retos que le quieren imponer.

La discusión alrededor de las medidas también puso en evidencia cómo detrás de los resultados macroeconómicos positivos se esconden contradicciones económicas graves que no garantizan el éxito de aquéllas ni propician la prosperidad que prometen. Las medidas anunciadas tienen una orientación muy distinta a la declarada formalmente, los datos en los cuales se fundamentan no son confiables, pues los asesores económicos los alteran de acuerdo a sus conveniencias, y, por último, las experiencias chilena, argentina y mexicana - guardadas las distancias debidas-cuestionan su fundamento real.

Estas contradicciones muestran la falta de una estrategia económica consistente, pero al mismo tiempo revelan el carácter aventurero de la nueva política. No es pura casualidad que ninguno de sus propugnadores se haya atrevido a garantizar su éxito. Cuando son preguntados sobre él, responden con evasivas. Si por alguna razón llegase a faltar el respaldo monetario necesario en dólares, el país no podrá maniobrar para contrarrestar la crisis y sus consecuencias serán catastróficas para todos. Si éste fuese el caso, ya no habria agricultura ni industria para superar el bache.

En definitiva, entonces, cómo se explica la adopción de una política económica tan controvertida y sin un apoyo social representativo. En primer lugar, como un tributo al mercado, a la globalización y a la apertura indiscriminada. El mercado y el crecimiento se presentan como las premisas básicas del bienestar social, pero se trata de unas premisas contradictorias $\dot{e}$ inconsistentes. Contradictorias porque al mismo tiempo que el mercado se expande y el producto nacional crece, la pobreza también se generaliza y se profundiza, el desempleo se globaliza y la sociedad se vuelve más violenta e insolidaria. La dinámica del mercado ha favorecido la concentración de bastantes sectores claves en formas de propiedad mono y oligopólicas. Inconsistentes porque los índices eco- 
nómicos que respaldan las premisas son manipulados de acuerdo a las conveniencias de quienes defienden la propuesta gubernamental.

En segundo lugar, se explica como una profundización de la política económica del gobierno anterior, cuya meta era promover las exportaciones. Ahora se busca favorecer la acumulación de capital de aquellos industriales, exportadores y comerciantes que, amparados por el gobierno de Cristiani, concentraron la riqueza nacional y se apoderaron de la banca privatizada, violando abiertamente la ley de privatización. Este selecto grupo de capitalistas es el único que se encuentra preparado para competir en el mercado internacional y para globalizar sus inversiones.

El siguiente paso parece consistir en apoderarse de los fondos de previsión social de diversas instituciones estatales, excepto los de la Fuerza Armada, que los necesita para participar plenamente en las oportunidades ofrecidas por la nueva política económica. De esta manera, el grupo privilegiado tendría acceso a unos fondos que le permitirían apoderarse de las comunicaciones y de los servicios de agua y energía, cuyas empresas son altamente rentables y lo serán mucho más si, además, interviene la inversión extranjera. La adquisición de esos fondos y de esas empresas no tendría mayor costo para estos capitalistas salvadoreños. Así, la llamada modernización del Estado, es decir, la privatización de las empresas estatales rentables, es un punto clave de las medidas anunciadas.

En conclusión, la nueva política económica está realmente orientada a expandir el capital y las ganancias de este reducido grupo de privilegiados, conformado en los últimos cinco años al amparo del primer gobierno de ARENA. La privatización y las medidas que la acompañan están orientadas a facilitar su participación en los mercados financieros internacionales. Por lo tanto, el único que se moderniza y se globaliza es' este exclusivo grupo de capitalistas que, además, ha conseguido el poder necesario para poner a su servicio al segundo gobierno de ARENA, no obstante la existencia de diferencias políticas a primera vista insalvables entre ambos grupos.

\section{Articular la política económica con la política social}

Los éxitos macroeconómicos también ocultan contradicciones sociales que fácilmente se convierten en un factor de inestabilidad e inseguridad. La presentación oficial de las medidas fue precedida por una enumeración de sus objetivos generales. Todos ellos se reducen a uno sólo, mejorar la calidad de vida de la población. En efecto, según el presidente de la república, las medidas se justifican porque ellas harán realidad la aspiración de los salvadoreños: convertir El Salvador en un país don- 
de predomine la equidad al haber igualdad de oportunidades para todos.

Lo que aún no se ha explicado, sin embargo, es cómo se llevará a cabo esa reconversión si las medidas únicamente ofrecen oportunidades a quienes se apoderaron de la banca, aprovechándose de su privatización. No se ha explicado cómo se llevará a cabo la función social que el presidente Calderón dice que la economía tiene, en particular, cómo las cuatro medidas, en lugar de seguir permitiendo que un reducido grupo de capitalistas privilegiados acumule más riqueza, la redistribuirán para mejorar el nivel y la calidad de vida de la población. Tampoco se ha explicado cómo estas cuatro medidas llevarán al bien común, eliminando lo que el presidente de la república llamó en su discurso "distorsiones", es decir, que unos pocos se hayan estado beneficiando de las políticas económicas gubernamentales en perjuicio de las grandes mayorías.

Más concretamente y siempre apoyados en la primpera parte del discurso presidencial (reproducido en la sección de "Documentación" de esta edición), conviene conocer cuánta riqueza va a generar esta nueva política, qué proporción de ella se invertirá en educación y salud, en mejorar las condiciones de vida en las zonas rurales y urbanas marginales, donde la pobreza se concentra, cómo fortalecerá a la pequeña y mediana empresa y cómo va a impedir la venta del bienestar de los trabajadores salvadoreños si entre sus planes se encuentra convertir el país en una gran zona franca, pues la esencia de la maquila es la sobreexplotación de la fuerza laboral, los salarios bajos, la violación de los derechos de los trabajadores y la escasa capacitación que éstos reciben y necesitan. Cuando los salarios suben y se aplican las leyes laborales, las maquiladoras abandonan las zonas francas y buscan otras donde el "ambiente" sea más favorable.

Ni el presidente de la república ni sus ministros han respondido a estas interrogantes de carácter social. El primero se limitó a hacer una lista de los beneficios genéricos que la población obtendría con la nueva política, dejando la explicación de los detalles a los segundos. Pero éstos todavía no han podido explicar concretamente las implicaciones sociales de las medidas económicas. La explicación es importante por dos razones. La primera, porque, en realidad, las medidas no están orientadas a eliminar la "distorsión" señalada por el presidente de la república, sino todo lo contrario, ésta aumentará al seguir privilegiando aún más a los mismos

\section{Estas contradicciones muestran la falta de una estrategia económica consistente, pero al mismo tiempo revelan el carácter aventurero de la nueva política.}


grandes capitalistas en perjuicio de la mayoría de la población. La segunda, porque, según el discurso presidencial, la nueva política económica sólo se justifica si se corrige esta distorsión y se elimina la pobre$z a$, de lo contrario, no tendría sentido.

A estas preguntas sólo se puede responder con realismo si la política económica se articula con la inversión social. Para lograrlo no es suficiente añadir adjetivos como integral, sostenible, sustentable y equitativo al sustantivo desarrollo. La articulación es difícil, pero urgente y necesaria, si en verdad se busca el bien común. La dificultad mayor radica en que hasta ahora el así llamado "desarrollo" se ha regido por las reglas de un capitalismo más bien voraz, por no decir salvaje, la máxima ganancia en el menor tiempo posible. Así, el "desarrollo" sólo comprende a los sectores privilegiados, opera con plazos cortos, depreda el medio ambiente y aumenta las desigualdades ya de por sí escandalosas. Es claro, entonces, que el simple juego de palabras no cerrará la brecha cada vez más ancha entre el lujo de unos pocos y la pobreza de la mayoría.

El riesgo social que la nueva política acarrea es tan grave que hasta las grandes gremiales de la empresa privada lo señalaron en el debate previo. Movidas más por el temor que por un interés social genuino, introdujeron en la polémica sobre las medidas valoraciones éticas al hablar de consumismo desenfrenado, provocado por la terciarización de la economía; de la mala calidad de la vida de las mayorías populares; de poner en peligro la estabilidad social y política por incrementar los indices macroeconómicos; de transparencia en el proceso de privatización; de la importancia de las remesas familiares para sostener la economía del país y de la necesidad de una relación armónica entre empleadores y trabajadores.

Indudablemente, en esta postura influye mucho la amenaza que la nueva politica económica implica para la sobrevivencia de la empresa privada tradicional, pero si ésta mantiene

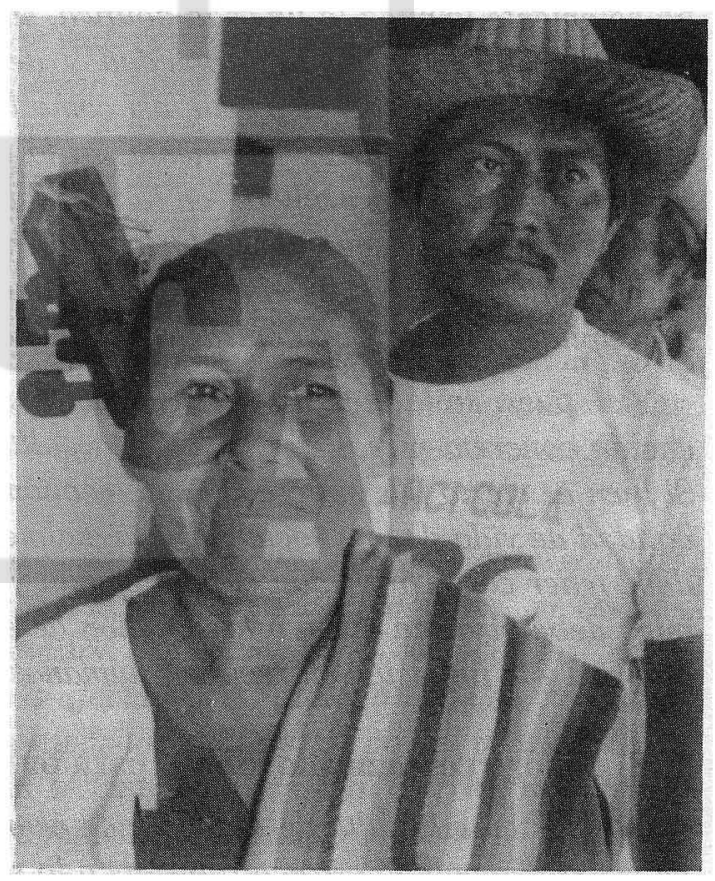


sus valoraciones éticas por encima de su interés particular, estas últimas podrían ser un buen punto de partida para replantear la estrategia económica del país. Los principios básicos de esta estrategia debieran ser la erradicación de la pobreza y la mejora de la calidad de vida de la población. Sin embargo, para que esto pueda ser posible, la empresa privada debe abandonar la postura más bien egoísta que hasta ahora ha mantenido frente a las necesidades de las mayorías populares. Ello implicaría conversiones éticas y sacrificios económicos y sociales importantes, entre ellos, una reducción de sus ganancias, de por sí ya escandalosamente elevadas.

Para los buenos salvadoreños y salvadoreñas, todo esto debiera ser obvio. Siempre se ha dicho que lo mejor de El Salvador es su población. Parece, pues, que ha llegado el momento de fortalecerla mejorando su nivel de vida. El verdadero salvadoreño es aquel que está dispuesto a sacrificar sus propios intereses por el bienestar de sus compatriotas. Es contradictorio que un país que se enorgullece de la tenacidad y del coraje de su población al mismo tiempo la mantenga hambrienta, enferma e ignorante. En la lucha contra la pobreza es donde se demuestra quién es verdadero salvadoreño. Las gremiales de la empresa privada parecen tener claro el problema y su vía de solución, sólo falta que se pongan manos a la obra y demuestren hasta dónde llega su capacidad para sacrificarse por los demás.

Ciertamente, sin democracia no es posible la paz, pero la democracia no solamente implica la libertad política, sino que, además, es contraria a la concentración de la propiedad y del capital. Ambas cosas son opuestas a una democracia real, así como lo es la dictadura militar o el régimen autoritario. Aparentemente, en lo único en lo que hay acuerdo hasta ahora es en lo primero, en la necesidad de la democracia para la paz, pero no en lo segundo. De hecho, la nueva política económica gubernamental parece desconocer que la concentración de la propiedad y del capital es totalmente contraria a la democratización de la sociedad.

Así, pues, en la política gubernamental se echa de menos una planificación concreta y real que articule la política económica con la social. Si bien es cierto que sin crecimiento económico no es posible mejorar la calidad de vida de una sociedad; es igualmente cierto que el crecimiento debe tener como prioridad el bien común y para ello, la política económica debe girar en torno a la creación de empleo, la erradicación de la pobreza y la inversión en recursos humanos.

\section{El diálogo como medio idóneo para buscar el bien común}

No obstante la falta de una política económica consistente e integral en cuanto vinculada estructuralmente a lo social y cultural, el presidente 
de la república inició un diálogo novedoso, antes de anunciar las nuevas medidas económicas. En efecto, por primera vez en la historia del país las medidas económicas son presentadas y discutidas ampliamente. Hasta ahora, lo normal era que el debate se desarrollara una vez que la política económica había sido anunciada. Más aún, hay que reconocer que el presidente de la república escuchó el debate y modificó algunos elementos importantes de las medidas: no elevó el impuesto al valor agregado y la desgra-

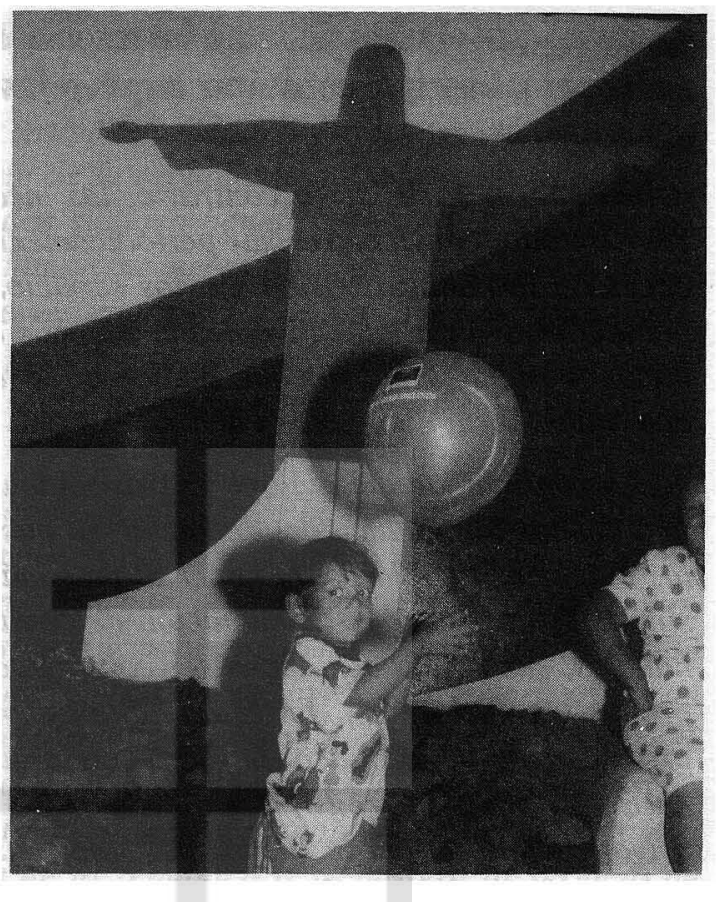
vación arancelaria será más lenta de lo previsto inicialmente. La consulta incluyó a los gobiernos de la región, puesto que la desgravación arancelaria tiene consecuencias en los tratados de integración vigentes.

El diálogo, sin embargo, fue limitado porque excluyó a la mediana, pequeña y micro empresa, a los sectores populares, a las iglesias y a los académicos; porque quienes fueron consultados por el presidente de la república nunca discutieron una estrategia de desarrollo, ni siquiera llegaron a considerar las cuatro medidas en su conjunto, sino que discutieron sobre cada una en particular; porque el gobierno no entregó los análisis en los cuales las medidas se fundamentan; porque no se hizo un diagnóstico serio sobre la situación económica y social del país. Para que el diálogo adquiera carácter nacional tanto por quienes toman parte en él como por la temática, estas limitaciones deben superarse. De todas maneras, se ha dado un paso importante a tener en cuenta en el futuro inmediato.

Y es que el diálogo debiera continuar porque, dado lo arriesgado de las medidas, una vez implementadas, sus resultados debieran ser revisados periódicamente y en esa revisión debieran participar todos los sectores sociales, sobre todo los más afectados. El diálogo debiera continuar también para verificar si los propósitos declarados por el presidente de la república sobre las medidas se cumplen, en particular los relacionados con lo social. Una vez aprobadas las medidas, no debe olvidarse que la erradicación de la pobreza y la igualdad de oportunidades para todos 


\section{Una vez que el mito de la no intervención del Estado se ha roto, hay que constatar a quién favorece con su actuación.}

deben conseguirse, de lo contrario, las medidas carecerían de sentido. Por lo tanto, conviene revisar periódicamente si el empleo aumenta, si el acceso a la canasta básica y a los servicios públicos se amplía, y si la población avanza educativamente. Además de discutir los temas económicos y sociales habría que incluir en el debate el tema cultural, del cual nada se ha dicho hasta ahora.

La continuidad del diálogo es trascendental porque, dado que la política económica anunciada, de hecho, no busca favorecer los intereses de las mayorías populares, ni hacer al país más productivo, ni más seguro, sino sólo favorecer la acumulación de capital para incrementar sus ganancias, sus resultados deben ser contrastados sistemáticamente con los propósitos declarados. En este sentido, debiera revisarse el proceso de privatización de la banca, llevado a cabo durante el primer gobierno de ARENA, para corregir y sancionar las violaciones de la ley de privatización. $Y$, desde luego, para que las futuras privatizaciones no caigan en vicios similares, el proceso debe ser conducido abierta y transparentemente.

Continuar el diálogo sería trascendental también como un ejercicio democrático permanente, en el cual tanto la sociedad como los políticos dijeran su palabra. Sin embargo, el diálogo no debe limitarse a los politicos porque éstos, por lo general, sólo representan los intereses de sus partidos y no necesariamente los de la sociedad. Una razón adicional para no reducir el diálogo a la clase política es que, en la actualidad, ésta se encuentra muy desprestigiada ante la población. Por eso, entre todos aún es posible encontrar los fundamentos y definir los lineamientos de una política económica, social y cultural verdaderamente nacional.

En esta nueva experiencia dialogante, el Estado es un factor determinante. Debe promover el diálogo para escuchar cuidadosamente lo que los ciudadanos tienen que decirle y para luego atender fielmente sus demandas. Es cierto que el gobierno de turno tiene un mandato popular, pero ese mandato no le da derecho para administrar el Estado a su antojo mientras dure el periodo constitucional establecido. Tampoco vale alegar que el Estado debe participar lo menos posible en la vida nacional, porque ya es inocultable que interviene cuando le conviene a los grupos que representa - por ejemplo, cuando compra dólares incurriendo en grandes pérdidas y alentando la inflación para mantener la tasa de cambio artificialmente elevada porque así conviene a los intereses de los grandes exportadores. 
Una vez que el mito de la no intervención del Estado se ha roto, hay que constatar a quién favorece con su actuación. Hasta ahora, por lo general, ha favorecido a los grupos económica y militarmente más poderosos. Entonces, el presidente de la república no debiera extrañarse que sólo un grupo de privilegiados se haya beneficiado de las políticas oficiales. Dado que el Estado interviene necesariamente, por qué no modificar a los beneficiarios de estas intervenciones; en lugar de favorecer a los más poderosos y ricos, por qué no empieza a privilegiar a los más pobres de los pobres. Las intervenciones estatales y sus destinatarios debieran formar parte de la agenda del diálogo nacional.

No se puede seguir confiando en supuestos beneficios que siempre se obtendrán en el futuro a cambio de aceptar políticas y modelos económicos que mientras enriquecen a los que ya son ricos y poderosos, empobrecen y debilitan a quienes ya son pobres y débiles. La profundización y extensión de la pobreza debe ser uno de los temas prioritarios del diálogo nacional que el presidente de la república debe continuar de forma sistemática. Desde luego, un diálogo de esta naturaleza impediría que el futuro del país cayera en manos de unos cuantos asesores con acceso directo al presidente de la república o se pusiera al servicio de unos intereses determinados y excluyentes. Sólo la búsqueda perenne del bien común puede asegurar y garantizar la vida de todas las salvadoreñas y de todos los salvadoreños.

San Salvador, 15 de febrero de 1995. 